\title{
Optical Character Recognition (OCR) System for English Language using Artificial Neural Network (ANN) Classifier
}

\author{
Honey Mehta ${ }^{1}$ and Sanjay Singla ${ }^{2}$
}

Abstract- Character recognition is a very complex task when one is talking about record keeping of all the data in digital format which can be further used to perform various manipulation operations. The actual problem is character recognition of various fonts in which the text is written. We proposed a new approach with the concept of Artificial Neural Network(ANN) for character recognition from scanned images. Once the data is implemented by an OCR(Optical Character Recognition) system and converted into digital form, it can be edited, manipulated and stored in computer understandable form.

Keywords - : Unicode, Artificial Neural Network, Scanned images

\section{INTRODUCTION}

Today, computers have become a dominant part of human's life. They tend to have a critical role in every field of medical science to production units. Growth of internet has lead to increase in the requirement for online information thus leading to increasing demand of cybernation of printed material for the purpose of searching and storing. Therefore character recognition systems are being developed for translating the human readable text to machine encoded form. Disregarding the faster calculations and operations, computers still lack behind humans in the area of problem solving. Thus, artificial neural networks were originally developed which mimics the behaviour of human nervous system to some extent [1]. Being the earliest application of artificial intelligence, ANN still lacks behind human interpretation of texts and images disregarding latest developments. Thus, improvement in character recognition systems for more coherent and resourceful results is highly required. In this paper we present a new approach using Artificial Neural Network to detect text from the scanned or inputted images. Non linearity of ANN assists with the complex nature of text recognition from input images. In the paper we proposed objectives to deal with the problem under consideration and then the methodology includes implementations. At the end, the performance of the system id observed by mathematical tables.

\section{CHARACTER RECOGNITION}

Character recognition is a process of identification of printed, typewritten or handwritten characters. The characters are optically scanned and converted into machine editable form for the purpose of storing and research.

Major uses includes-

- Form of data entry for old books

\footnotetext{
${ }^{1}$ Department of Computer Science Engineering IET Bhaddal, Ropar, Punjab,India
}

${ }^{2}$ Department of Computer Science Engineering IET Bhaddal, Ropar, Punjab,India 
- Storage of office papers, bank forms

- Storing old and decaying material like handwritten manuscripts for research purposes

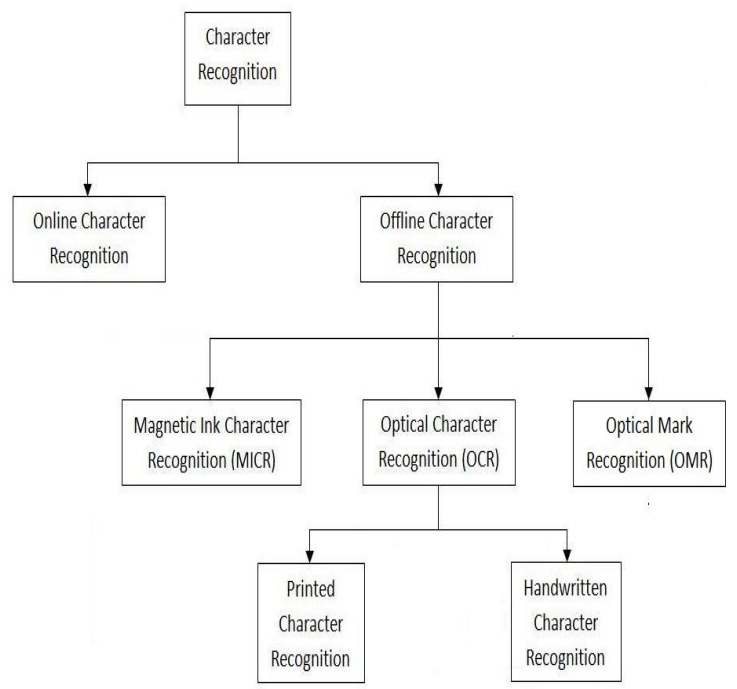

Fig. 1. Types of character recognition

Due to difference in usage and source of writing of certain texts, the character recognition process generally falls under two categories:

- Offline character recognition

- Online character recognition

Online character recognition refers to the real time acquirement and perception of characters. An optical pen is used for writing characters and recognition is done in parallel with writing and the results are displayes on a screen.It is also called handwriting recognition as it recognizes the characters written by hand through optical pen.

Offline character recognition refers to the recognition of characters present on a sheet of paper. The paper is first scanned by a digital equipment like scanner or camera and then the image is stored in the computer which is further used in the process of recognition of specific characters.

Offline character recognition is divided into three categories-

- Magnetic Ink Character Recognition (MICR)

- Optical Character Recognition (OCR)

- Optical Mark Recognition (OMR)

\section{ARTIFICIAL NEURAL NETWORKS}

Many soft computing techniques are used in recent days by many researchers to perform such tasks where there is no such requirement of hard computing and the solution space is very large. Artificial neural network is also one of the soft computing solution which these days we are using. This is one of the basic solutions a particular problem ever have. The main factors which forces us to use these kind of techniques are listed as:

- POWER- the power of neural network helps to find the solutions from large solution domains by concentrating on acknowledgements only. 
- EASY TO USE- In training phase data collection step is used to collect all related information in the network about the solution and the at the step of interpretation this knowledge is use for decision making process.

The main purpose of ANN is to act as a classifier for various classification algorithms. The basic inspiration behind ANN is the biological neuron system in the brain of humans. How neurons in the brain of human act as a processing units and share information to generate results. Intelligent computer systems can be made on the basic of ANN also by giving sufficient amount of training to the system.

\section{The Basic Artificial Model-}

The definition of ANN and its process is given below:

- The inputs are provided to the neurons by using the original data according to the specific application. Weights are assigned to each input coming to the neuron. The basis of these neurons is the synaptic efficiency or the capability of the neurons. Different threshold values are also designated with different neurons. After counting the weighted sum which generates an input to the neurons, the output of the neuron is calculated by the application of suitable activation function.

- Activation function are used to generate the output at the output neuron. One of the activation function is bipolar activation function which generate output either as -1 or 1 .

In this paper, we propose an for character recognition using Multi-Layered percepton (MLP) ANN system. The proposed structured is a three layer architecture. First is the input layer which receives input in the form of segmented character images of accepted size. Second layer is a hidden layer which is use to train the neural network regarding specific font styles in case of character recognition process. The final layer is the output layer which is used to generate Unicode values for different characters which itself worked as a matching criteria.

\section{RELATED WORK}

Apurva Srivastav and Jayant kumar [1] proposed a system for text detection. The focused on the constraint about the width of the stroke which form a character. The proposed the technique which can work on different font style and size. Also the background shade and text orientation issues were addressed by the authors. In this text regions with weak edges can be captured with average of $96.38 \%$ recall and $79.21 \%$ precision.

Junsik at el. [2] developed a system where the wine label images were firstly captured by the mobile camera and then text present on label was recognized. Noise and irregular font issues were addressed by the authors. For the purpose of classification authors used fuzzy c-means clustering and shape based feature.

JingSong at el. [3]. proposed a safe CAPTCHA framework. They considered the Multiple Moving Object Recognition in complex background problem. The difficulty level of the problem domain produced the need of high precision classifier for the proposed system.

Shangqin at el. [4]. proposed a sytem to detect license plate numbers using OCR. The focused on the concept of Wavelet transform to deal with the texture features. Authors separate the color information for the recognition purpose. The proposed system worked well in complex backgrounds but not able to locate in case of black backgrounds because of the issue of IHLS color space.

S. Benson at el. [8]. proposed a system go generate image CAPTCHA which prevent the security system from automatic web tools meant for the destruction of CAPCHA systems. They focused on the 
issue of segmentation at character level, shape matching during classification process and blind attack while handling security issues.

Rong-Chi at el. [11]. presented text detection algorithm from natural scene images. Their proposed algorithm follow to steps as: Firstly, Canny edge detector was used to find candidate regions. Secondly, a fast connected component (CC) algorithm was used to obtain text. Authors achieved $94.65 \%$ precision rate for text extraction.

Based on the above related work some of the identified problems are addressed in the next section.

\section{PROBLEM DEFINITION}

Work done in this field of text detection is mainly performed by using gradient features. Some of the researchers use color segmentation approach to the analysis of text.

The only assumption that the existence of text in large gradient regions may affect some applications where the scenario is reversed

These algorithms only considers that pixels with large gradient values can be considered under text area.

$>\quad$ Various edge detection methods were presented in the literature. all are based on luminance only. Color segmentation may be required to produce good results.

\section{OBJECTIVES}

- A database of characters will be formed by collecting a wide range of scanned printed alphabets(A-Z).

- $\quad$ Input image will be de noised and enhanced using pre-processing step.

- Image processing using basic filtering

- Feature extraction using artificial neural networks.

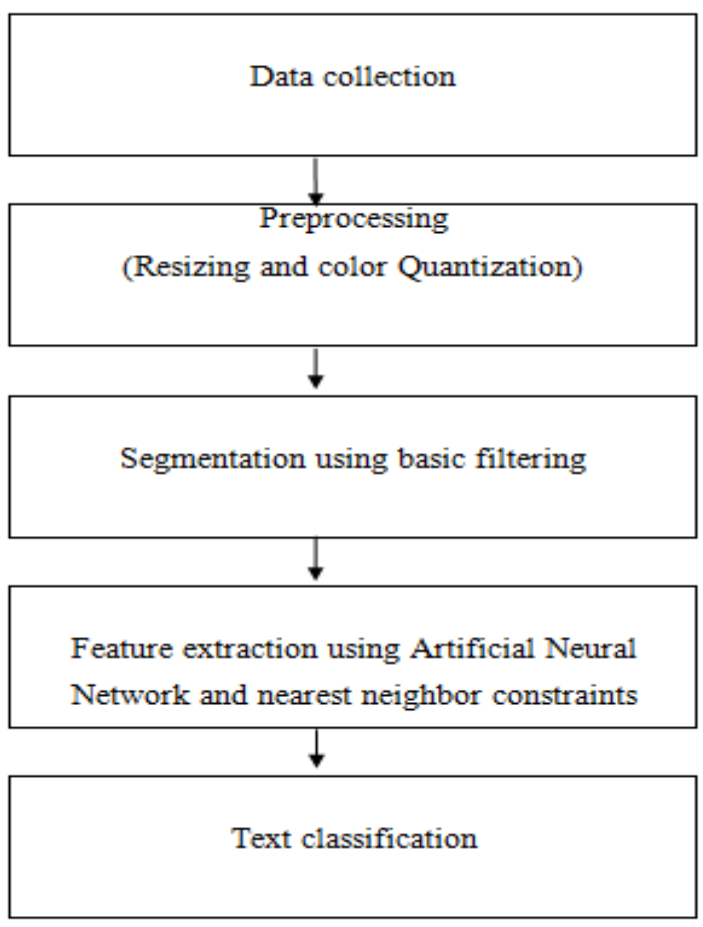

Fig2. Methodology used 
- Text classification using nearest neighbour constraints and verification based on different parameters like false positive detection, false negative detection, recall and precision.

\section{PROPOSED SYSTEM}

We proposed a system here for recognition of characters from images with the help of Artificial Neural Network and nearest neighbour approach.

The main parts of the consideration are the data collection, pre processing step, then the extraction of symbol of interest from the input scanned image, after that conversion of this identified or located symbol into a pixel matrix of specific or standard size. Then the next part is the classification of the symbol under consideration which can be done by assigning the different loads to the layer of neural network. In case of data collection, the hidden layer neurons have to assign weights for the classification of characters according the specific font style.

Nearest Neighbour Approach: It is used for classification purposes .Here, for every component, two consecutive nearest neighbours are found by the application of Euclidean distance. The different parameters like dimensions, distance and the alignment of component and their neighbours are compared.

\section{Algorithm for classification of characters}

Form network according to the specified topology parameters

1. Fix the number of layers of the network and also fix the number of neurons in each layer.

2. Assign weights to the different neurons. Also set the biased value for each output neuron.

3. Initialize the hidden layer by providing desirable data from training set file.

4. Input the image and identify and place different character symbols in a liner array.

5. Formation of output is done at output layer with the help of Unicodes.

6. For every character symbol feed forward output is collected for further use.

7. Now the Error computation can be performed using error function.

8. Adjust weights according to the error using the concept of back propagation.

9. Repeat steps 6 to 8 for other characters too.

10. Averaging of error can be computed for all characters.

11. Up to fix number of epoch repeat step 6 to step 10.

\section{RESULTS AND DISCUSSIONS}

Performance of our system is observed by considering the variations in number of iterations and the variations in the number of characters. The proposed system produce $98.89 \%$ recognition rate for two different fonts by considering up to 90 characters at a time.

Here , Font style 1= latin arial 
Font style 2= latin tahoma

Table 1. Variations in number of epochs

\begin{tabular}{|c|c|c|c|c|c|c|}
\hline $\begin{array}{c}\text { Number } \\
\text { of } \\
\text { Epoch }\end{array}$ & \multicolumn{2}{|c|}{$\mathbf{3 0 0}$} & \multicolumn{2}{c|}{600} & \multicolumn{2}{c|}{800} \\
\hline $\begin{array}{c}\text { Font } \\
\text { Styles }\end{array}$ & $\begin{array}{c}\text { No. of } \\
\text { mismatch }\end{array}$ & $\begin{array}{c}\text { Error } \\
\text { Percentage }\end{array}$ & $\begin{array}{c}\text { No. of } \\
\text { mismatch }\end{array}$ & $\begin{array}{c}\text { Error } \\
\text { Percentage }\end{array}$ & $\begin{array}{c}\text { No. of } \\
\text { mismatch }\end{array}$ & $\begin{array}{c}\text { Error } \\
\text { Percentage }\end{array}$ \\
\hline $\mathbf{l}$ & 04 & 04.44 & 03 & 03.33 & 01 & 01.11 \\
\hline $\mathbf{2}$ & 01 & 01.11 & 0 & 0 & 0 & 0 \\
\hline
\end{tabular}

Here, Number of chars $=90$ sigmoid slop $=0.014$

Table 2: Variations in number of input characters

\begin{tabular}{|c|c|c|c|c|c|c|}
\hline Number & \multicolumn{2}{|c|}{20} & \multicolumn{2}{|c|}{50} & \multicolumn{2}{|c|}{90} \\
\hline $\begin{array}{l}\text { Font } \\
\text { Styles }\end{array}$ & $\begin{array}{c}\text { No. of } \\
\text { mismatch }\end{array}$ & $\begin{array}{c}\text { Error } \\
\text { Percentage }\end{array}$ & $\begin{array}{c}\text { No. of } \\
\text { mismatch }\end{array}$ & $\begin{array}{c}\text { Error } \\
\text { Percentage }\end{array}$ & $\begin{array}{c}\text { No. of } \\
\text { mismatch }\end{array}$ & $\begin{array}{c}\text { Error } \\
\text { Percentage }\end{array}$ \\
\hline 1 & 0 & 0 & 06 & 12 & 11 & 12.22 \\
\hline 2 & 0 & 0 & 03 & 06 & 08 & 8.89 \\
\hline
\end{tabular}

\section{CONCLUSION}

In this paper a new method of classification of characters from image containing text is presented with the help of MLP neural network and Nearest Neighbor constraint approach. Following are the points concluded under this research work.

Non-Linear nature of ANN is good for the complex problem like character recognition.

$>$ Recognition rate of proposed method is $98.89 \%$, which is considerable one.

$>$ Segmentation process produce near to $100 \%$ results also.

\section{REFERENCES}

[1] Srivastav,A., Kumar,J.,2010, "Text Detection in Scene Images using Stroke Width and Nearest-Neighbor Constraints"

[2] Lim,J., Kim,S., Park,J., Lee,G., Yang,H., Lee,C.,2009, "Recognition of Text in Wine Label images"

[3] Cui,J., Wang,L., Mei,J., Zhang,D., Wang,X., Peng,Y., Zhang,W.,2010, “CAPTCHA Design Based on Moving Object Recognition Problem"

[4] Mao,S., Huang,X., Wang,M.,2010, “An Adaptive Method for Chinese License Plate Location “

[5] Kumar,M., Kim,Y.C., Lee,G.S.,2010, "Text Detection using Multilayer Separation in Real Scene Images"

[6] Al-Shamma,S.D., Fathi,S.,2010,“Arabic Braille Recognition and Transcription into Text and Voice"

[7] Ikica,A., Peer,P.,2011,"An improved edge profile based method for text detection in images of natural scenes"

[8] Raj,S.B.E., Devassy,D., Jagannivas,J.,2011, “A New Architecture for the Generation of Picture Based CAPTCHA"

[9] Wazalwar,D., Oruklu,E., Saniie,J.,2011, "Design Flow for Robust License Plate Localization” 
[10] Shivakumara,P., Phan,T.Q., Shijian Lu, Tan.C.L.,2011,"Video Character Recognition Through Hierarchical Classification"

[11] Chang,R.C.,2011,"Intelligent Text Detection and Extraction from Natural Scene Images"

[12] Zhang,S., Li,H.,2011, "Video Semantic Mining based on Dense Sub Graph Finding"

[13] Oi-Mean Foong, Hairuman,I.F.B., 2011, "OCR Signage Recognition with Skew \& Slant Correction For Visually Impaired People"

[14] Wang,X., Lai,W., 2010, "Edge Detection for Chinese Text Image Based on Novel Differential Operator"

[15] Huang,X., 2012, “Automatic Video Text Detection and Localization Based on Coarseness Texture"

[16] Bo Bai, Fei Yin, Cheng-Lin Liu ,2012,“A Fast Stroke-Based Method for Text Detection in Video"

[17] Shih-Chung Chen, Chung-Min Wu, Shih-Bin Su, 2012,"Image Morse Code Text Input System"

[18] Mello,C.A.B., Costa, D.C., Santos,T.J.D., 2012, "Automatic Image Segmentation of Old Topographic Maps and Floor Plans"

[19] Zhang,Y., Wang,C., Xiao,B., Shi,C., 2012, “A New Text Extraction Method Incorporating Local Information"

[20] Yang,Z., Shi,P.,2012,“Caption Detection and Text Recognition in News Video”

[21] Rajeshbaba, M., Anitha, T., 2013, "Detect and Separate localization Text in Various Complicated-Colour Image" 